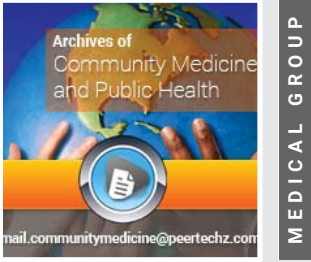

\title{
Early evidence for effectiveness of statewide school closure for mitigation of influenza
}

\section{Jonathan L Temte ${ }^{1 *}$, Cecilia $\mathrm{He}^{2}$ and John Tamerius ${ }^{2}$}

'Department of Family Medicine and Community Health, School of Medicine and Public Health, University of Wisconsin in Madison, WI 53715, USA

${ }^{2}$ Quidel Corporation in San Diego, CA 92121, USA
Received: 26 May, 2021

Accepted: 15 June, 2021

Published: 16 June, 2021

*Corresponding author: Jonathan L Temte, MD, PhD Department of Family Medicine and Community Health, School of Medicine and Public Health, University of Wisconsin in Madison, WI 53715, USA, E-mail: jon.temte@fammed.wisc.edu

Keywords: School closure; Influenza; Nonpharmaceutical interventions; Primary carec https://www.peertechzpublications.com

\section{Check for updates}

\section{Abstract \\ Background: School closures were mandated in response to COVID-19 as a nonpharmaceutical intervention. Influenza A was widespread in Wisconsin at the time of mandate, March 18, 2020, allowing its evaluation for influenza interruption.}

Methods: Daily percent positivity of influenza A among primary care patients who received a rapid influenza diagnostic test was evaluated throughout two influenza seasons while schools were open and closed.

Results: Median deviation from baseline of percent positivity was significantly lower when schools were closed than when open $(-2.25 \%$ vs $1.08 \%$; $\mathrm{H}=7.84 ; \mathrm{P}=0.005)$. Median deviation in percent positivity for the eight days following statewide school closure was -20.0 .

Discussion: Unprecedented closures of all Wisconsin schools was associated with a rapid decline in influenza A. Additional evaluations within other jurisdictions are warranted.

\section{Introduction}

School closure is an important component of Nonpharmaceutical Interventions (NPI) for pandemic influenza [1]. Given similarities in transmission dynamics between SARSCoV-2 and influenza, school closure was implemented rapidly as a countermeasure for COVID-19 across the United States [2] (Figure 1) and elsewhere. Wisconsin enacted statewide, mandated closure of public and private schools commencing on March 18, 2020. Many school districts, however, did not open on March 16, 2020, effectively making closure effective after Friday, March 13, 2020.

Throughout early March, our surveillance team was monitoring rising rates of influenza $\mathrm{A}(\mathrm{H} 3 \mathrm{~N} 2)$ following an unusual year in which Wisconsin had experienced successive waves of influenza B and influenza $\mathrm{A}(\mathrm{H} 1 \mathrm{N1})[3]$. Evaluation of medically attended, laboratory-confirmed influenza (MAI), through a Centers for Disease Control and Prevention program [4], was hampered, however, by rapidly changing approaches to care in surveillance clinics during the week of March 9, 2020. We were able to continue our influenza surveillance efforts using a surveillance system based on automated reporting of

Number of K-12 Students Affected by School Closure (United States, March 16, 2020 through April 2, 2020)

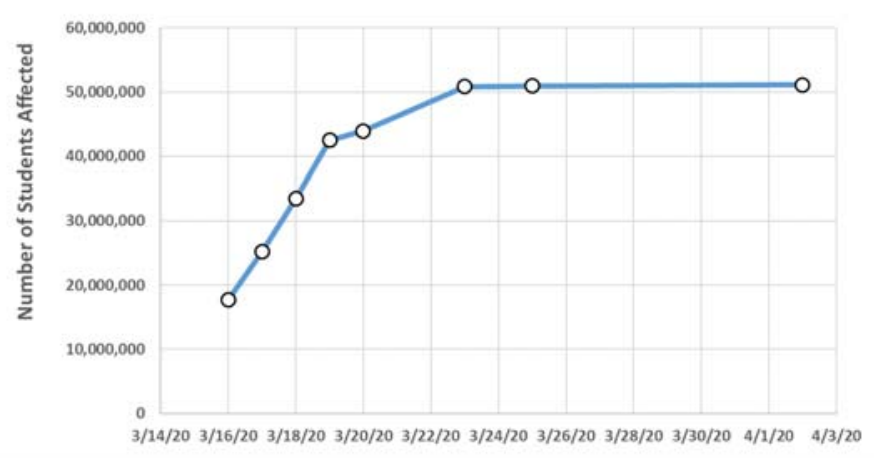

Figure 1: Timing of K-12 school closures and number of students affected in the United States during March and April, 2020. 
influenza A and B results from rapid influenza diagnostic tests in primary care practices [5].

The widespread and abrupt closure of schools across Wisconsin provides an opportunity to evaluate the potential benefit of proactive school closure for SARS-CoV-2 on MAI [6]. Whereas school closure is considered an important NPI for mitigating the effects of pandemic influenza [1], less evidence exists for its role in controlling outbreaks of seasonal influenza. The objective of this study was to assess the potential role of school closures on an independent marker of seasonal influenza across two academic years and ending with widespread school closures across one state.

\section{Methods}

We used data from Quidel's Virena surveillance platform [7] to estimate daily prevalence of influenza across Wisconsin starting on August 1, 2018 through March 26, 2020. This system is able to aggregate all reported rapid influenza diagnostic tests (RIDT) across an entire state in near real-time. Because of variability in the number of tests performed per day, and due to pandemic-related changes in clinic visitation rates in early March 2021, we used the percent positivity as a marker for influenza A prevalence [8]. The percent positivity is less likely to be affected by patterns of patient attendance than the number of positives as it corrects for specimen volume. We limited our school closure analyses to two periods conforming to the usual season of influenza circulation in Wisconsin: November 1, 2018 through April 30, 2019 and November 1, 2019 through March 26, 2020.

We used the academic calendar of the Oregon School District (OSD: Oregon, Wisconsin) as a proxy for typical school closures across Wisconsin. There is high concordance on the timing of the autumn (Thanksgiving) and winter break (Christmas through New Year's) across Wisconsin [9]. Less concordance exists for the timing of spring break and shorter breaks. Of note were multiple short closures during January and February, 2019 due to extremely cold conditions across the upper Midwest. All schools in Wisconsin underwent long-term closure starting between March 14 and March 18, 2020. School closure for this study was defined as any weekday on which school was closed during the study periods. Weekend days were included only if they were continuous with a weekday closure. This allowed a separation from "usual" school attendance and "altered" due to closure. In addition, a weekend that is continuous with one or more closure day(s) can potentially interrupt influenza transmission due to serial interval of influenza [10].

We compared the daily percent positivity of influenza A to a preceding 21-day baseline for percent positivity, with a one week washout period. Accordingly, we summed the number of positive tests over the three week period, occurring from 28 to 8 days before the day of interest, and divided by the total tests performed during this period to provide the baseline. We then subtracted the baseline estimate from the daily estimate of percent positivity to assess the deviation from the baseline.
Because deviations from baseline were not normally distributed, we compared the median deviations from baseline for school closure days to non-closure days using the KruskalWallis statistic. In addition, we introduced a five-day lag to assess potential delayed effects of reduced transmission in school settings, thus allowing for one to two serial intervals of influenza [10]. Finally, we assessed the mean deviation from baseline of the last nine days of data following the statewide school closure for SARS-CoV-2 mitigation.

\section{Results}

Over the entire surveillance period, 15,093 influenza tests were reported yielding an average of 25.0 results per day (range: $0-143$ per day). There were 2,789 positive test results for influenza A with an overall percent positivity of $18.5 \%$. There were no significant differences in the daily percent positivity rate based on day of the week $\left(\mathrm{F}_{(6,597)}=0.41 ; \mathrm{P}=0.871\right)$.

Across the two study periods, there were 80 school closure days out of a total of 327 days ( $24.5 \%)$. Variability existed in the daily deviation from baseline of the percent positivity (Figure 2) with three notable periods of decline associated with the winter and spring breaks during the 2018-2019 school year and with the statewide school closure in 2020.

The median deviation from baseline was significantly lower when schools were closed than when open (median $=-2.25 \%$ vs. $1.08 \% ; \mathrm{H}=7.84 ; \mathrm{P}=0.005)$. This relationship was preserved following introduction of a five-day lag to allow for potential effects on influenza transmission (median $=-2.29 \%$ vs. $1.34 \%$; $\mathrm{H}=12.63 ; \mathrm{P}<0.001)$. The mean and median deviation in percent positivity for the eight days following statewide school closure were-19.2 \pm 4.1 and -20.0 , respectively.

\section{Discussion}

A statistically significant reduction in the percent positivity of Rapid Influenza Diagnostic Tests (RIDT) for influenza A was noted in the days following a statewide closure of Wisconsin schools in response to the SARS-CoV-2 pandemic. This reduction, as measured by a network of clinics using RIDT

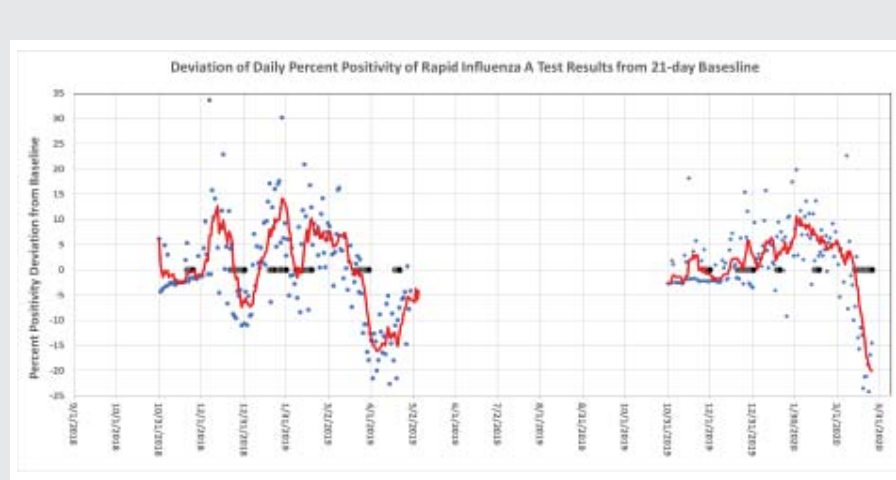

Figure 2: Deviation of daily percent positivity of rapid influenza diagnostic tests results for influenza A from 21-day baseline with 7-day washout period (blue dots) in Wisconsin during two influenza seasons (2018-2019 and 2019-2020). The seven day moving average is depicted by the red line. School closure days are depicted by black dots placed on zero line. One outlying data point with $100 \%$ positivity (one positive test one for one test performed) on December 24, 2019 is not shown.

Citation: Temte JL, He C, Tamerius J (2021) Early evidence for effectiveness of statewide school closure for mitigation of influenza. Arch Community Med Public Health 
suggests that statewide school closure is potentially a potent mitigating strategy for influenza A. Moreover, periods of school closure, when coincidental to influenza circulation, appear to be linked to reductions in MAI.

During the 2018-2019 school year, a year dominated by influenza A, most influenza A in Wisconsin did not occur until after mid-February 2019. In 2019-2020, influenza A was in low circulation across Wisconsin before early January [3]. Consequently, declines in percent positivity seen in December 2018 and February 2019 may be of less relevance than those noted in March of 2019 coinciding with spring break and in March 2020 coinciding with mandated school closure.

This assessment has major limitations. First, there is no direct linkage between MAI and school-aged children. The findings are coincidental, but not necessarily causative. Second, clinical behavior was in significant flux starting in mid-March 2020 due to the SARS-CoV-2 pandemic, and thus, could alter estimates based on rapid influenza diagnostic testing at primary care clinics. Third, we used the OSD as a proxy for school districts across Wisconsin. This could introduce some error into the timing of short school breaks and for spring break in the 2018-2019 academic year. Fourth, the profound declines occurring coincidentally with late season closures occurs at the time of "usual" declines in influenza prevalence, and may introduce some confounding. Finally, school closure in Wisconsin came shortly before the "Safer at Home" emergency order, issued on March 24, 2020 [11]. Consequently, behavioral changes preceding this formal order may have had effects on influenza transmission.

Although there is a large body of literature on the role of school closure in reducing influenza spread, there is a lack of consensus on its effectiveness. During the 2009 influenza A(H1N1) pandemic, district level closures in Michigan were found to have little effect on ILI [12], while a study in Japan found school closures reduced the number of infected students by $24 \%$ at its peak [13]. These closures have also shown to reduce contact patterns, suggesting capability to mitigate influenza spread $[14,15]$. There are further controversies and limited evidence for the timing and length of closures [6], thus making inferences about the precise impact of school closure challenging.

School closure represents an extreme form of social distancing within a young population with high likelihoods of person-to-person transmission of respiratory viruses. As most states mandated school closure during the week of March 16,2020 [2], an excellent opportunity exists to evaluate the roles of school closure on transmission of influenza and other respiratory viruses. Such assessment, however, may fail to elucidate potential impacts of school over small geographical range and over varying time frames. We therefore encourage additional evaluations of the potential effects of school closure taking into account both the scale (statewide, regional and local) and timing (scheduled vs. unscheduled; proactive vs. reactive) of closure.

\section{Implications for policy \& practice}

Nonpharmaceutical interventions, including school closure, are essential elements of pandemic influenza response.

Unprecedented school closures due to SARS-CoV-2 present a unique opportunity to examine their effects on seasonal influenza detection rates within communities. We took advantage of an existing surveillance system; others may be able analyze similar data to determine the effects of statewide school closure on the level of influenza.

Planned and unplanned school closures may reduce the number of influenza cases in communities. This information could help public health experts and school administrators develop protocols for strategically-timed school breaks to mitigate influenza and other respiratory viruses.

\section{Financial and Other Disclosures}

This study was part of Ms. He's MPH capstone project at the University of Wisconsin School of Medicine and Public Health. Dr. Temte has received past research support from Quidel Corporation.

\section{Acknowledgements}

We thank Quidel Corporation for providing Wisconsin influenza A test result data from Virena and Cristalyne Bell for assisting with manuscript preparation.

Human Participant Compliance Statement: No IRB approval was needed for this project using only anonymous test result data from an existing registry.

\section{References}

1. Centers for Disease Control and Prevention (2017) Community mitigation guidelines to prevent pandemic influenza - United States, 2017. MMWR Recommendations and Reports 66: 1-32. Link: https://bit.ly/3iGTb6m

2. Education Week (2020) Map: Coronavirus and School Closures in 2019-2020 Link: https://bit.ly/3wtMbht

3. Wisconsin State Laboratory of Hygiene (2020) Influenza Activity. Link: https://bit.ly/3pXalhC

4. Fowlkes A, Steffens A, Temte J, Lonardo SD, McHugh L, et al. (2015) Influenza Incidence Surveillance Project Working Group. Incidence of medically attended influenza during pandemic and post-pandemic seasons through the Influenza Incidence Surveillance Project 2009-13. Lancet Respir Med 3: 709718. Link: https://bit.ly/2RXgZbf

5. Temte JL, Barlow S, Schemmel A, Temte E, Hahn DL, et al. (2017) New method for real time influenza surveillance in primary care: A Wisconsin Research and Education Network (WREN) supported study. J Am Board Fam Med 30: 615623. Link: https://bit.ly/3gADVFB

6. Viner RM, Russell SJ, Croker H, Packer J, Ward J, et al. (2020) School closure and management practices during coronavirus outbreaks including COVID-19: a rapid systematic review. Lancet Child Adolesc Health 4: 97-404. Link: https://bit.ly/3xjNKyv

7. Quidel Corporation. Virena - Global Wireless Surveillance and Remote Data Management. Link: https://bit.ly/3iJnPMo

8. World Health Organization (2012) WHO Interim Global Surveillance Standards for Influenza. Link: https://bit.ly/3zwToPQ 
9. Temte JL, Meiman JG, Gangnon RE (2019) School sessions are correlated with seasonal outbreaks of medically attended respiratory infections: electronic health record time series analysis, Wisconsin 2004-2011. Epidemiol Infect 147: e127. Link: https://bit.ly/2Ssl1c7

10. Cowling BJ, Fang VJ, Riley S, Malik Peiris JS, Leung GM (2009) Estimation of the serial interval of influenza. Epidemiology 20: 344-347. Link: https://bit.ly/3xhvmWS

11. Wisconsin Department of Health Services (2020) Emergency Order \#12. Safer at Home Order. Link: https://bit.ly/3xmhguf

12. Davis BM, Markel H, Navarro A, Wells E, Monto AS, et al. (2015) The effect of reactive school closure on community influenza-like illness counts in the state of Michigan during the $2009 \mathrm{H} 1 \mathrm{~N} 1$ pandemic. Clin Infect Dis 60: e90-e97. Link: https://bit.ly/2RZkRZq
13. Kawano S, Kakehashi M (2015) Substantial impact of school closure on the transmission dynamics during the pandemic flu H1N1-2009 in Oita, Japan. PLoS One 10: e0144839. Link: https://bit.ly/3cKAnzn

14. Litvinovaa M, Liuc Q-H, Kulikove ES, Ajellia M (2019) Reactive school closure weakens the network of social interactions and reduces the spread of influenza. Proc Natl Acad Sci U S A 116: 13174-1381. Link: https://bit.ly/35pkn1C

15. Tamerius J, Viboud C, Shaman J, Chowell G (2015) Impact of school cycles and environmental forcing on the timing of pandemic influenza activity in Mexican states, May-December 2009. PLoS Comput Biol 11: e1004337. Link: Link: https://bit.ly/2U3yo2F
Discover a bigger Impact and Visibility of your article publication with

Peertechz Publications

\section{Highlights}

* Signatory publisher of ORCID

* Signatory Publisher of DORA (San Francisco Declaration on Research Assessment)

* Articles archived in worlds' renowned service providers such as Portico, CNKI, AGRIS, TDNet, Base (Bielefeld University Library), CrossRef, Scilit, J-Gate etc.

* Journals indexed in ICMJE, SHERPA/ROMEO, Google Scholar etc.

* OAI-PMH (Open Archives Initiative Protocol for Metadata Harvesting)

* Dedicated Editorial Board for every journal

* Accurate and rapid peer-review process

* Increased citations of published articles through promotions

* Reduced timeline for article publication

Submit your articles and experience a new surge in publication services (https://www.peertechz.com/submission).

Peertechz journals wishes everlasting success in your every endeavours.

Copyright: () 2021 Temte JL, et al. This is an open-access article distributed under the terms of the Creative Commons Attribution License, which permits unrestricted use, distribution, and reproduction in any medium, provided the original author and source are credited. 